\title{
Zwischen Wahrheit und Richtigkeit
}

\section{Sozialpädagogische Forschung in der Dialektik objektiver und subjektiver Ansprüche}

\author{
Bernd Birgmeier und Martin Stummbaum
}

PD Dr. Bernd R. Birgmeier ist Akademischer Rat am Lehrstuhl für Sozialpädagogik und

Gesundheitspädagogik an der Katholischen Universität EichstättIngolstadt.

E-Mail bernd.birgmeier@ku-eichstaett.de Martin Stummbaum ist wissenschaftlicher Mitarbeiter am Lehrstuhl für Sozialpädagogik und Gesundheitspädagogik an der Katholischen Universität Eichstätt-Ingolstadt. E-Mail: martin.stummbaum@ku-eichstaett.de
Soziale Arbeit als Wissenschaft und Praxis benötigt sowohl Grundlagenforschung als auch anwendungsbezogene Forschung.

Die Wissenschaftstheorie verpflichtet jedes Fachgebiet dazu, (mindestens) vier Voraussetzungen zu erfüllen, um als autonome Wissenschaft anerkannt zu werden. Diese Voraussetzungen - oftmals auch »Organisationselemente « von Wissenschaftsdisziplinen genannt - verpflichten auch die Soziale Arbeit darauf

- sich auf einen Gegenstand zu beziehen

- Erkenntnismethoden zu benennen

- Theorien zu entwickeln und

- zu forschen (vgl. Engelke 2003).

Das zuletzt genannte Element, die Forschung, nimmt dabei einen hervorgehobenen Stellenwert innerhalb dieser Kriterienquadriga ein, weil sie einerseits logische und vermittelnde Bezüge zwischen den anderen Organisationselementen herstellt, andererseits deren eigentlichen Funktionen überhaupt erst ermöglicht.

Mit anderen Worten: Erstens bleibt ein Gegenstand (Objektbereich) einer Disziplin so lange im Dunkeln, bis er nicht durch Forschung - »erhellt « wird; zweitens können Theorien erst durch die präoder posttheoretische Funktion der Forschung entwickelt werden; und schließlich - drittens - ist die Benennung (und Anwendung) von Erkenntnismethoden selbst nichts anderes als die Entscheidung für (oder gegen) einen bestimmten Forschungszugang zum Objekt. Nicht von ungefähr gilt Forschung deshalb als Bürge wissenschaftstheoretischer Anforderungen und als das Kennzeichen schlechthin der Wissenschaft(-lichkeit) einer Disziplin.

So unbestreitbar die elementare Bedeutung von Forschung für die Wissenschaft(en) auch ist: Das Prädikat der »wissenschaftlichen « Forschung offenbart zugleich jedoch Unklarheiten, die unmittelbar in einem nur schwer eindeutig abzugrenzenden Begriff der »Wissenschaft « selbst liegen. Forschung - als das
Herzstück von Wissenschaft - wird demnach in einen ähnlichen diffusen Begriffsund Definitionsstrudel hineingezogen, gegen den die Wissenschaft seit jeher anzukämpfen hat. Wenn wir dementsprechend der Formel »Ohne Forschung keine Wissenschaft, keine Wissenschaft ohne Forschung! « nachgehen wollen, ist daher zu bedenken, dass sich im Laufe der Jahrhunderte viele Wissenschaften immer mehr ausgegliedert haben, und Wissenschaft als solche einer stetig fortlaufenden Spezialisierung und rapide ablaufenden Zellteilung anheim gefallen ist (vgl. Ströker 1994). Solche Entwicklungen führen aktuell dazu, dass der Wissenschaftsbegriff einerseits ins nahezu Monströse anschwillt, während er andererseits - etwa durch Fragen nach der Nutzbarkeit von Erkenntnissen - ins Beliebige seiner Verwendung abzugleiten droht.

Aufgrund der Beliebigkeit und Offenheit der Verwendung des Wissenschaftsbegriffes gerät die Suche nach »wahren « Erkenntnissen heutzutage in ein weit verzweigtes Koordinaten-System von Dialektiken, mit denen - einmal mehr - die Frage nach dem Subjekt-Objekt-Verhältnis zu stellen ist.

Diesen Gedanken nach der Polarität zwischen Subjekt und Objekt koordinatorisch weiter geführt, stoßen wir auf mindestens zwei klärungsbedürftige Achsen, deren Verbindung das Fadenkreuz der Forschung auszumachen scheint: Eine erste Achse skizziert forschungsmethodologische Pole, die nach Bedingungen der Möglichkeit forschenden Zugriffs auf einen Objektbereich (Gegenstand) generell fragen und damit nach Voraussetzungen des objektiv erklärenden und beschreibenden sowie des subjektiv verstehenden »Wissen Schaffens « innerhalb disziplinärer Forschungstraditionen. Eine zweite Achse fokussiert demgegenüber unmittelbar die Rolle (und den Einfluss) des jeweiligen Subjekts im Forschungsprozess und - darüber - die Individualität und Subjektivität des Forschers und des Be- 
forschten. Um dieses Fadenkreuz der Forschung - mit dem auch (subjektiv) abhängige und (objektiv) unabhängige Variablen der Forschung zur Diskussion gebracht werden - in ein heuristisches Orientierungsraster einzubetten, bedienen wir uns im Folgenden den übergeordneten Begriffen und Lesarten der Objektivität und Subjektivität von Forschung und den ihnen zugrunde liegenden Besonderheiten und Mechanismen.

\section{Objektbezogene Lesarten der Forschung}

Orientieren wir uns - im Sinne einer Arbeitsformel - an Forschung als »systematische, d. h. theoretisch und methodologisch begründete und überprüfbare Beobachtung, Beschreibung und Rekonstruktion gesellschaftlicher Ausschnitte der Wirklichkeit auf der Basis sozial-wissenschaftlicher Erhebungs- und Analyseverfahren « (Lüders/Rauschenbach 2001, 562), so wird deutlich, dass mit dieser Definition mehrere wissenschaftstheoretische Aspekte differenziert werden müssen.

Einmal wichtig ist hier die Beachtung der unterschiedlichen methodologischen Wege, die Forscher im Sinne der Erkenntnisgewinnung gehen müssen. Je nach dem, was der Wissenschaftler konkret zu dem in Frage stehenden Gegenstand wissen möchte, wählt er aus diversen Ansätzen philosophischer Positionen der Wissenschaftstheorie diejenige Erkenntnismethode, die eine Durchdringung seiner spezifischen Frage an den Gegenstand am ehesten gewährleistet. Dabei kann er - im Spiegel klassischer Erkenntnis-Methodik - empiristisch, rationalistisch, pragmatisch, phänomenologisch, positivistisch, konstruktivistisch, hermeneutisch etc. vorgehen (vgl. Carrier 2006, Poser 2006, Chalmers 2007).

In Abhängigkeit zu diesen vielfältigen methodologischen Zugängen haben sich im Verlauf der Wissenschaftsgeschichte einzelne Wissenschaftsformen und Wissenschaftsarten mit unterschiedlichen erkenntnistheoretischen Präferenzen herausgebildet, mit denen auch eine Systematisierung von Wissenschaft(en) möglich wird. So lassen sich Disziplinen entweder nach ihrem genuinen Gegenstandsgebiet - also Wissenschaften vom Menschen, von der Gesellschaft, von der Kultur etc. - klassifizieren (vgl. Schurz 2006); oder man orientiert sich an Grenzziehungen, wie wir sie aus der Unterscheidung zwischen nomothetischen und idiographischen, zwischen $\mathrm{Na}$ tur- und Kulturwissenschaften, zwischen Sozial- und Geisteswissenschaften kennen. Besonders die zuletzt genannte Einteilung in Sozial- und Geisteswissenschaften zwingt die Forscher dazu Stellung zu nehmen, ob sie ihr Objekt (oder Teile davon) erklären oder verstehen (bzw. subjektiv deuten, interpretieren) wollen. sondern um Nützlichkeit der Forschung. Damit obliegt das Erkenntnisinteresse nicht mehr (nur) wissenschaftstheoretischen Kriterien, sondern oftmals ausschließlich denjenigen, die sich einen wie auch immer gearteten - subjektiven Nutzen (Status, Prestige, finanzielle Mittel etc.) von der Forschung versprechen (vgl. Lüders/Rauschenbach 2001). Auftraggeber dieser Art von Forschung können Institutionen, Organisationen, Verbände, Kommunen, politische Gemeinschaften, Einzelpersonen sein, die sich zuvörderst an ihren Zielen orientie-

\section{»Forschung spielt sich immer zwischen wissenschaftlichen und persönlichen Interessen $a b \ll$}

Gleichgültig, welcher Intention und Funktion nun Folge geleistet wird: Allen methodologischen Zugängen der (empirischen) Sozialforschung - sowohl in sämtlichen ihrer qualitativen als auch in ihren quantitativen Ausprägungen - gemein ist die Forderung, hierüber objektives, also nachprüfbares und empirisch gesichertes Wissen und "Wahrheiten « zu einem Objekt zu generieren. Dies gilt auch - trotz der Kritik so mancher strenger (Sozial-) Wissenschaftler - für hermeneutische und (re)konstruktivistische Forschungszugänge, in denen das Subjekt (also der Mensch) zum Objekt erhoben wird.

\section{Subjektbezogene Lesarten der Forschung}

Während die oben ausgeführte Lesart der Forschung - hinlänglich auch mit dem Begriff »Grundlagenforschung « etikettiert - zum ersten die Objektivität auf doppelte Weise in den Mittelpunkt ihres Erkenntnisinteresses rückt (einmal die Objektivität ihrer Resultate und zum zweiten die Objekt-Bezogenheit ihres $\mathrm{Zu}$ griffs), werden in einem weiteren Zweig, der sogenannten anwendungsbezogenen Auftrags- oder Bedarfsforschung, objektive Kriterien vielfach durch subjektive Forschungsaspekte untergraben. Hier geht es also nicht (nur) um Wahrheit, ren und daher all das in das Gewand von Forschung packen, was (subjektiv) brauchbar und nützlich ist.

Nicht selten werden daher subjektive Bedürfnisse und Motive Einzelner zu den wahren Beweggründen und Leitlinien forschenden Tuns und konterkarieren das, was eine an der Objektivität orientierte erkenntnisbezogene Grundlagenforschung der » reinen Wahrheit « wegen und im Dienste der Wissenschaft unternimmt. Solche - bisweilen gefährlichen - Randerscheinungen im Forschungsgeschäft verdeutlichen einmal mehr, dass Forschung und Wissenschaft zu den kulturellen Produkten der Menschheit zählen und damit (spezifische) menschliche Tätigkeiten beschreiben, die eben von Menschen vollzogen werden, die sich (allzu!) menschlich und damit mitunter fehlerhaft und nicht immer ethisch-moralisch korrekt verhalten. Wie in allen Bereichen sozialer Realität ist somit auch Forschung und ihr Anspruch, objektiv Erkenntnisse zu gewinnen koabhängig von subjektiven Faktoren, wie beispielsweise der Persönlichkeitsstruktur, das familiale (soziale) Umfeld, die sozio-ökonomische Lebenssituation des Forschers etc. (vgl. Engelke 2003).

Jenseits dieser (subjektiven) Gefahrenmomente gibt es jedoch - analog zur objektbezogenen Lesart - auch eine positive Kehrseite subjektbezogener Forschung, 
die dann deutlich wird, wenn wir unseren Blick nicht (nur) auf den Akteur, sondern auf den Adressaten richten, der nicht nur von außen (passiv) erforscht wird, sondern der bisweilen auch selbst aktiv am Forschungsprozess teilnimmt. Dies vor allem dann, wenn wir uns in das weite Feld qualitativer Forschungsmethodik begeben und uns auf dessen Programm, nämlich das Sinnverstehen und die Rekonstruktion sozialer und individueller Prozesse konzentrieren.

Im Zentrum dieser Forschungstradition steht das Subjekt und dessen individueller Lebens(ver)lauf, seine Biografie, Sinnkonstruktionen und Prozessstrukturen; dies alles kann über Interviews, teilnehmende Beobachtung etc. unmittelbar mit, durch und über das Subjekt erschlossen werden. Der Beforschte wird - indem er sich selbst beobachtet und beobachten lässt - zum (Ko-)Forscher, ohne den Erkenntnisgewinnung nicht möglich ist. Besonders deutlich wird dieser adressatenbezogene Moment subjektbezogener Forschung in der Aktionsforschung. Einzelne Subjekte sind dabei nicht bloß eine Informationsquelle für den Forscher, sondern Individuen, mit denen sich der Forscher gemeinsam auf den Weg der Erkenntnis macht (vgl. Burghardt 1998).

\section{Fazit}

Im Fadenkreuz der Forschung stehen sich Subjekt und Objekt (und damit abhängige und unabhängige Variablen) vielfach unvermittelt gegenüber, denn: Erkennen geschieht zwischen Subjekt und Objekt unter Einfluss wissenschaftstheoretischer und persönlich-subjektiver Interessen.

Ob das spannungsgeladene Verhältnis zwischen beiden Polen jemals hinreichend gelöst werden kann, bleibt abzuwarten; auch dann, wenn es bereits Synthetisierungsmodelle gibt (z. B. objektive Hermeneutik oder historische Bildungs- und Sozialisationsforschung), die beiden Seiten Rechnung tragen wollen. Und dennoch: Ein radikaler Konstruktivismus, der Form und Inhalt des Erkannten ausschließlich auf das Subjekt bezieht, ist aus der Forschung in Sozialer Arbeit jedenfalls ebenso wenig wegzudenken wie ein Realismus, Ontologismus oder kritischer Rationalismus, denen zufolge das Erkenntnisobjekt unabhängig vom Er- kenntnissubjekt existiert. Beide Bezugsgrößen haben ihre Berechtigung, aber auch ihre Grenzen.

Ein Forschungs-Anarchismus (Feyerabend), mit der ein "anything goes « propagiert wird und durch die ausschließlich den subjektiven Bedürfnissen von Forschern Rechnung getragen wird, scheint jedenfalls keine Lösung zu sein. Auch eine rein von materiellen und kommerziellen Zielen getriebene, sogenannte anwendungsbezogene Wissenschaft lässt Objektivität und Universalität vermissen und gleitet meist in Parteilichkeit und forschungsethisches Versagen (vgl. Carrier 2006).

Nichtsdestotrotz ist Wissenschaft längst schon Teil der Selbstschöpfung von Forschern geworden und in viel stärkerem Umfang subjektiven Kräften unterworfen als je zuvor. Als Fazit ist daher festzuhalten: Ein Entweder-oder zum Problem des Subjekt-Objekt-Verhältnisses in der Forschung ist entweder wirklichkeitsfern oder egoistisch. Die Lösung liegt - wie in so vielen Bereichen menschlicher Realität - irgendwo dazwischen; idealiter in dem, was wir gemeinhin Verantwortung und professionelle Haltung nennen.

\section{Literatur}

Burghardt, T. (1998): Aktionsforschung. In: Huppertz, N. (Hg.): Theorie und Forschung in der Sozialen Arbeit. Neuwied.

Carrier, M. (2006): Wissenschaftstheorie. Hamburg.

Chalmers, A.F. (2007): Wege der Wissenschaft. Berlin.

Engelke, E. (2003): Die Wissenschaft Soziale Arbeit. Freiburg im Breisgau.

Lüders, Chr./Rauschenbach, Th. (2001): Sozialpädagogische Forschung. In: Otto, H.-U./Thiersch, H. (Hg.): Handbuch Sozialarbeit Sozialpädagogik. Neuwied. S. 562-575.

Poser, H. (2006): Wissenschaftstheorie. Stuttgart.

Schurz, G. (2006): Einführung in die Wissenschaftstheorie. Darmstadt.

Ströker, E. (1994): Probleme der Bestimmung und Abgrenzung von Wissenschaft. In: EuS 5/1994, S. 423-432.

\section{Das Berater- handbuch}

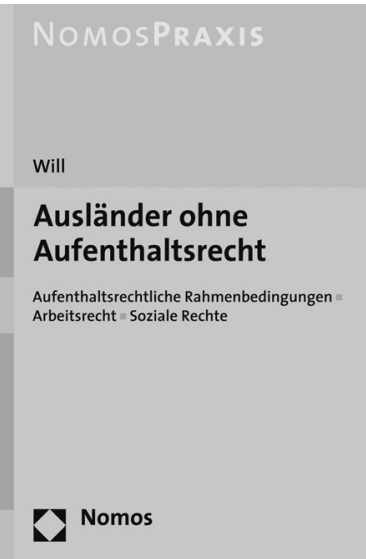

\section{Ausländer ohne \\ Aufenthaltsrecht}

Aufenthaltsrechtliche

Rahmenbedingungen /

Arbeitsrecht / Soziale Rechte

Von RAin Prof. Dr. Annegret Will

2008, 296 S., brosch., 39,- $€$

ISBN 978-3-8329-2500-O

Für die Beratung von Ausländern, die kein Aufenthaltsrecht (mehr) oder nur ein prekäres Aufenthaltsrecht besitzen, sind Kenntnisse des Aufenthalts-, Arbeits- und Sozialrechts ebenso unentbehrlich wie straf- und datenschutzrechtliches Wissen. Das Handbuch behandelt sämtliche Rechtsfragen, die sich in der Beratungsarbeit stellen.

\section{Nomos}

Bitte bestellen Sie im Buchhandel oder versandkostenfrei unter - www.nomos-shop.de 